\title{
COVID-19 Infection: Viral Clearance and Antibody Response in Dialysis Patients and Renal Transplant Recipients
}

\author{
Paolo Ferdinando Bruno ${ }^{a}$ Maria Cappuccilli ${ }^{b}$ Alessandra Spazzoli ${ }^{a}$ \\ Matteo De Liberali ${ }^{c}$ Brunilda Sejdiu $^{d}$ Marianna Napoli $^{b}$ Vera Minerva $^{b}$ \\ Simona Semprini ${ }^{e}$ Giorgio Dirani ${ }^{\mathrm{e}}$ Vittorio Sambrie Andrea Buscaroli ${ }^{\mathrm{d}}$ \\ Angelo Rigotti ${ }^{c}$ Elena Mancini ${ }^{f}$ Paolo Masperi ${ }^{g}$ Gaetano La Manna ${ }^{b}$ \\ Giovanni Mosconi ${ }^{\mathrm{a}}$
}

${ }^{a}$ Nephrology and Dialysis Unit, AUSL Romagna Morgagni-Pierantoni Hospital, Forlì, Italy; ${ }^{b}$ Department of Experimental Diagnostic and Specialty Medicine (DIMES), Nephrology, Dialysis and Renal Transplant Unit, S. Orsola-Malpighi Hospital, University of Bologna, Bologna, Italy; 'Nephrology and Dialysis Unit, AUSL Romagna

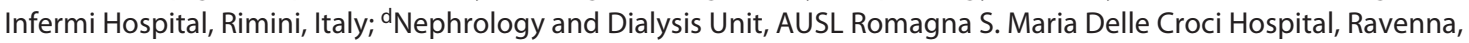
Italy; ${ }^{\text {eUnit }}$ of Microbiology, AUSL Romagna Laboratory Pievesestina Cesena (FC), Cesena, Italy; ${ }^{f}$ Nephrology, Dialysis and Hypertension Unit, S. Orsola-Malpighi Hospital, Bologna, Italy; ${ }^{9}$ Medical Direction Unit, AUSL Romagna Morgagni-Pierantoni Hospital, Forlì, Italy

\section{Keywords}

Chronic renal failure · Coronavirus disease $2019 \cdot$ Dialysis

Kidney transplantation · Viral clearance

\begin{abstract}
Background/Aims: The coronavirus disease 2019 (COVID-19) pandemic is the major current health emergency worldwide, adding a significant burden also to the community of nephrologists for the management of their patients. Here, we analyzed the impact of COVID-19 infection in renal patients to assess the time to viral clearance, together with the production and persistence of IgG and IgM antibody response, in consideration of the altered immune capacity of this fragile population. Methods: Viral clearance and antibody kinetics were investigated in 49 renal patients recovered from COVID-19 infection: 7 of them with chronic decompensated renal failure, 31 under dialysis treatment, and
\end{abstract}

11 kidney transplant recipients. Results: The time span between the diagnosis of infection and recovery based on laboratory testing ( 2 negative nasopharyngeal swabs in consecutive days) was $31.7 \pm 13.3$ days. Three new positive cases were detected from 8 to 13 days following recovery. At the first serological determination after swab negativization, all the patients developed IgG and IgM antibodies. The semiquantitative analysis showed a progressive increase in IgG and a slow reduction in IgM. Discussion/Conclusion: In subjects with decompensated chronic kidney disease, under dialysis and in transplant recipients, viral clearance is lengthened compared to the general population. However, in spite of their common status of immunodepression, all of them were able to produce specific antibodies. These data might provide useful insights for monitoring and planning healthcare activities in the weak category of patients with compromised renal function recovered from COVID-19.

(c) 2021 S. Karger AG, Basel karger@karger.com

www.karger.com/nef

Karger'

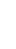

(C) 2021 S. Karger AG, Basel 


\section{Introduction}

The coronavirus disease 2019 (COVID-19) pandemic is a major clinical and social problem in all the countries. At the end of year 2020, the number of cases worldwide was $83,102,166$, with $1,812,671$ deaths and $58,897,317$ recovered (https://www.worldometers.info/coronavirus/, browsed on December 31, 2020).

The impact on health care in the nephrological setting is significant. In analogy with previous infections by other coronaviruses [1], COVID-19 has shown a significant spread among patients with advanced chronic renal failure (stages 3b, 4, and 5 based on the Chronic Kidney Disease Epidemiology Collaboration [CKD-EPI] classification), those under chronic dialysis treatment and kidney transplant recipients $[2,3]$. The infection is associated with poorer outcomes in subjects with impaired renal function than in the general population, mainly due to the presence of concurrent chronic diseases, in particular cardiovascular comorbidity [4]. Moreover, besides the well-known link of SARS-CoV-2 infection and respiratory distress syndrome, there are further potential mechanisms leading to systemic multiorgan failure. The reason lies in the wide distribution of angiotensin-converting enzyme 2 receptor, the gate of entrance of the virus. Thus, in case of a high viral load, the infection can spread through the angiotensin-converting enzyme 2 receptor to various target organs, including the kidney, heart, liver, brain, endothelium, gastrointestinal tract, immune cells, and erythrocytes (thus causing thromboembolism) [5-7].

The mortality rate reported in the national survey data of the Italian Society of Nephrology is $33.76 \%$ in patients receiving hemodialysis treatment and $24.77 \%$ in renal transplant recipients [8]. Akin to the general population, death risk is higher with increasing age. Current evidence supports individual inflammatory response as a key player in the development of the disease with its related complications. The immunodepression induced by the metabolic status of chronic kidney disease (CKD) and dialysis [2] or by post-transplant antirejection therapy [3] can influence the clinical course and the eradication of SARSCoV-2 infection. To date, the role of advanced CKD or renal transplantation on viral clearance and on the pattern of IgM and IgG antibody response over time is still poorly understood [9-13].

A better awareness of the timing until SARS-CoV-2 negativization is particularly relevant for the possible management of organizational repercussions in different health-care settings. In hemodialysis units, the artificial replacement treatment is carried out until laboratory re- covery ( 2 negative nasopharyngeal swabs in consecutive days) in dedicated areas $[3,7,14]$. Similarly, in transplanted patients, the regular follow-up monitoring is conditioned by the persistence of the infection. Studies related to the viral load over time can represent a useful decisionmaking tool in case of infection relapse after the first recovery. Moreover, a full comprehension of the pattern of antibody production and persistence might also provide valuable indications for prophylaxis choices, above all vaccination, also in view of the known poor response to vaccination observed in patients with advanced CKD.

This study was undertaken to analyze the impact of COVID-19 infection in patients with chronic decompensated renal failure, those under dialysis treatment, and renal transplant recipients. In particular, we investigated the time to achieve viral clearance (recovery based on 2 negative nasopharyngeal swabs in consecutive days), as well as the kinetics of IgG and IgM antibody response, in consideration of the altered cellular and humoral immune responses commonly found in these patients.

\section{Materials and Methods}

\section{Patients}

A retrospective multicenter observational study was performed in chronic nephropathic patients who experienced SARS-CoV-2 infection (nasopharyngeal swab positivity regardless of subjective symptoms). The observation period was from February to April 2020, with data collection in May 2020.

The analysis considered patients followed at the Unit of $\mathrm{Ne}$ phrology and Dialysis of the local health authority of Romagna (Forli-Cesena, Ravenna, and Rimini) and at Nephrology, Dialysis, and Renal Transplant Unit of the S. Orsola Malpighi University Hospital of Bologna, an area with a total reference population of 2.2 inhabitants. Specifically, renal patients who recovered from COVID-19 infection (2 consecutive negative swabs) with the following clinical and functional characteristics were included:

1. Advanced chronic renal failure (KDOQI stages 3B-5) in conservative therapy with worsening renal function during infection and subsequent need for artificial replacement support (group 1).

2. Chronic dialysis treatment at the time of infection (group 2).

3. Clinically stable renal transplant received at least 6 months prior to the beginning of the study (group 3 ).

The time span between the first positive swab and the laboratory negativization was evaluated in all the subjects who met the above-mentioned inclusion criteria. The following general and clinical parameters were recorded: age, gender, estimated glomerular filtration rate (eGFR) based on CKD-EPI creatinine-based equation [15], dialysis vintage for group 2, and age at transplant for group 3.

The COVID-19-related data collected for this analysis were onset of the symptoms, severity of lung involvement, therapy for SARS-CoV-2 infection, and modulation of immunosuppressive 
therapy (for transplanted patients). Ethics Committee approval and informed consent were waived due to the observational nature of the study. The study followed the principles of the Declaration of Helsinki. All individuals cannot be identified in the study, as they have been fully anonymized.

\section{Laboratory Assays}

Nasopharyngeal swabs were performed according to the guidelines of the Italian National Institute of Health (Istituto Superiore di Sanità - ISS) [16]. Biological specimens were manipulated using appropriate personal protective equipment and at biosafety level 2, based on the recommendations by the World Health Organization and the European Centre for Disease Prevention and Control [17].

In all the swabs received at the laboratory of our Microbiology Unit, the presence of SARS-CoV-2 was assessed using a commercially available one-step real-time PCR (Allplex 2019-nCoV assay, Seegene, Seoul, South Korea) in which, after RNA extraction, retrotranscription and PCR amplification are carried out consecutively in the same reaction tube. This is a qualitative analysis based on the Seegene MuDT ${ }^{\mathrm{TM}}$ technology (combining $\mathrm{DPO}^{\mathrm{TM}}$ and $\mathrm{TOCE}^{\mathrm{TM}}$ ), which allows identification of multiple Ct values for every pathogen in each channel of the real-time PCR instrument. The target genes for the identification of SARS-CoV-2 are E (a specific gene common to Sarbecoviruses), RdRp, and N gene both specific for SARS-CoV-2. Sample positivity was attributed in case of detection of at least one of the 2 specific genes. The samples were extracted using the automated Microlab STARlet IVD platform (Hamilton Italia, Agrate Brianza, MB, Italy) and amplified using CFX96 Real-time PCR Detection System (CFX Manager SoftwareIVD v1.6) (Bio-Rad Laboratories, Segrate, Milan, Italy). The sensitivity declared by the manufacturer is 100 copies/reaction [18].

From 3 to 7 days following nasopharyngeal swab negativization, SARS-CoV-2 IgM and IgG antibody serum levels were measured using a solid-phase ELISA (COVID-19 IgG Enzyme ImmunoAssay and COVID-19 IgM Enzyme ImmunoAssay, DIA PRO Diagnostic Bioprobes, Milan, Italy), in line with the regional plan on serological tests. The assay identifies antibody binding to 3 virus-specific immunodominant antigens: nucleocapsid, spike 1 protein, and spike 2 protein. The detection of IgM antibodies requires a pretreatment step to remove the rheumatoid factor, as previously described [19]. The diagnostic sensitivity is $98 \%$. Serological semiquantitative determinations of IgG and IgM were carried out in a time span ranging from 3 to 8 days following negativization.

The IgG index was considered as negative if $<0.9$, uncertain (gray zone) if ranging between 0.9 and 1.1 , and positive if $>1.1$. The IgM index was considered as negative if $<0.9$, uncertain (gray zone) if ranging between 0.9 and 1.1 , and positive if $>1.1$. The C.I. for IgG index between 1.1 and 3.0 and for IgM index between 1.1 and 2.5 are quite low. These values were considered as weakly positive.

\section{Statistical Analysis}

Data are given as means \pm standard deviation for continuous variables and percentage and absolute numbers for categorical variables. Comparisons between continuous variables were made through Student's $t$ test or the nonparametric Mann-Whitney U or ANOVA followed by Tukey $t$ test, as appropriate. $\chi^{2}$ test was used to evaluate the categorical variables. A $p$ value below 0.05 was considered as significant, and all the statistical analyses were performed using the statistical package for the social sciences (SPSS ${ }^{\mathrm{TM}}$ for Windows Software Package, version 9.0.1; Chicago, IL, USA).

\section{Results}

This observational study considered a total of 75 renal patients diagnosed for COVID-19 in the period between February and April 2020, 9 of them with chronic decompensated renal failure (group 1), 50 on hemodialysis treatment (group 2), and 16 with a kidney transplant (group 3). There were no cases of infection in patients under peritoneal dialysis.

The incidence of COVID-19 was 3.8\% (50/1,303) in patients on chronic hemodialysis treatment and $3.5 \%$ $(50 / 1,423)$ if calculated on the total number of patients on renal replacement therapy (hemodialysis + peritoneal dialysis). In transplanted patients, the incidence was $1.7 \%$ (16/917). It was not possible to evaluate the incidence in patients with chronic decompensated renal failure due to the unavailability of the number of patients with eGFR $<45 \mathrm{~mL} / \mathrm{min}$ in the reference population.

Forty-nine out of 75 patients recovered from the infection. Overall mortality was $34.6 \%$ (26/75), specifically $22.2 \%(2 / 9)$ in group $1,38.0 \%(19 / 50)$ in group 2 , and $31.2 \%(5 / 16)$ in group 3.

Table 1 describes the main general and clinical features of the 49 patients who recovered from COVID-19 divided into patients with chronic decompensated renal failure (group 1, $n=7$ ), patients under chronic dialysis treatment at the time of infection (group 2, $n=31$ ), and renal transplant recipients (group 3, $n=11$ ). Gender distribution was comparable in 3 groups. On the other hand, pairwise post hoc comparisons revealed that kidney transplant recipients were significantly younger than the patients under hemodialysis $(p<0.05)$.

In the 7 patients of group 1, the baseline eGFR value was $31.1 \pm 15.3 \mathrm{~mL} / \mathrm{min}$ (CKD-EPI formula), then after COVID-19 infection, the worsening renal function required artificial replacement support for a period of 20.8 \pm 7.6 days (range 11-32 days). In group 2, all the 31 patients were on hemodialysis treatment. In groups 1 and 2, the artificial replacement treatments were performed in dedicated rooms (isolated from the conventional Dialysis Center) with continuous or intermittent standard techniques, depending on the specific situation. In group 3, the 11 transplanted patients had a preinfection eGFR of $47.7 \pm 25.4 \mathrm{~mL} / \mathrm{min}$, and they were predominantly male and younger than the other groups. 
Table 1. Main general and clinical features of the 49 patients who recovered from COVID-19

\begin{tabular}{lcccc}
\hline & $\begin{array}{l}\text { Advanced CKD } \\
\text { (group 1) }\end{array}$ & $\begin{array}{l}\text { Dialysis } \\
\text { (group 2) }\end{array}$ & $\begin{array}{l}\text { Renal transplant } \\
\text { (group 3) }\end{array}$ & Total \\
\hline$N$ & 7 & 31 & 11 & 49 \\
Age, years & $64.0 \pm 20.4$ & $70.4 \pm 14.9^{*}$ & $59.2 \pm 10.9^{*}$ & $67.0 \pm 15.5$ \\
Gender, M (\%) & $4(57.1)$ & $21(67.7)$ & $8(72.7)$ & $33(67.3)$ \\
Transplant age, months & - & - & $113.5 \pm 1120.0$ & - \\
Dialysis vintage, months & - & $19.8 \pm 16.7$ & - & - \\
\hline
\end{tabular}

The patients were divided into 3 groups: patients with chronic decompensated renal failure (group 1), patients under chronic dialysis treatment at the time of infection (group 2), and renal transplant recipients (group 3). Continuous variables are presented as means \pm standard deviation, and categorical variables as absolute numbers and percentage in brackets. Continuous variables were analyzed by ANOVA followed by Tukey's $t$ test for pairwise comparisons, and categorical variables by $\chi^{2}$ test. COVID-19, coronavirus disease 2019; CKD, chronic kidney disease. ${ }^{*} p<0.005$.

Table 2. Reasons for performing the nasopharyngeal swab in the 3 groups

\begin{tabular}{llll}
\hline Reason for swab sampling & $\begin{array}{l}\text { Advanced CKD } \\
\text { (group 1, } n=7)\end{array}$ & $\begin{array}{l}\text { Dialysis } \\
\text { (group 2, } n=31)\end{array}$ & $\begin{array}{l}\text { Renal transplant } \\
\text { (group 3, } n=11)\end{array}$ \\
\hline Clinical & $6(85)$ & $23(75)$ & $11(100)$ \\
Contact with a positive subject & $1(15)$ & $5(15)$ & $0(0)$ \\
Sporadic cases & $0(0)$ & $3(10)$ & $0(0)$ \\
\hline
\end{tabular}

Data are given in absolute numbers with the percentage in brackets. CKD, chronic kidney disease.

At the time of COVID-19 diagnosis, 8 patients ( 1 in group 1, 5 in group 2, and 2 in group 3 ) had respiratory symptoms (cough and upper respiratory tract inflammation) without related radiological lesions; 33 patients (6 in group 1, 18 in group 2, and 9 in group 3 ) had bilateral interstitial pneumonia. Only 8 patients, all of them in group 2, were asymptomatic.

Table 2 shows the reasons for performing the nasopharyngeal swab in the 3 groups, which were clinical (fever or respiratory disorders, even minor), close contact with a positive patient (only in groups 1 and 3 ), or sporadic cases (only 3 out of the 31 hemodialysis patients of group 2). After the diagnosis of COVID-19, all the patients were initially hospitalized (range of hospitalization length: 3-58 days), due to comorbidities related to chronic renal failure and/or immunodepression for the transplant recipients.

The treatment interventions were based on the experience of the center. During hospitalization, 5 patients, all of them in group 2, did not require oxygen therapy. In the remaining 44 patients, varying degrees of respiratory failure was reported: in 20 of them ( 3 in group 1, 13 in group
2 , and 4 in group 3$)$, low-flow oxygen therapy $(<6 \mathrm{~L} / \mathrm{min})$ was delivered using nasal cannulas or simple face masks, 18 ( 2 in group 1, 12 in group 2, and 4 in group 3) were treated with noninvasive ventilation by continuous positive airway pressure, and/or high-flow nasal cannulas, and 6 ( 2 in group 1, 1 in group 2, and 3 in group 3 ) required tracheal intubation and invasive mechanical ventilation.

The majority of the patients $(95 \%)$ received different therapy schemes over time based on antiviral agents (hydroxychloroquine, darunavir/ritonavir, or lopinavir/ ritonavir) and/or with tocilizumab and azithromycin. The distribution of the different drug regimes in the 3 groups are depicted in Table 3.

In all the transplant recipients under triple-drug immunosuppression regimen, mycophenolate or mTOR were withdrawn; also in 3 patients, calcineurin inhibitors were suspended during COVID-19 infection. Conversely, steroid therapy was always maintained or even enhanced.

The overall timing until SARS-CoV-2 negativization (documented by 2 consecutive negative swabs) in the to- 
Table 3. Therapy administered to the patients in the 3 groups

\begin{tabular}{llll}
\hline Therapy & $\begin{array}{l}\text { Advanced CKD } \\
\text { (group 1, } n=7)\end{array}$ & $\begin{array}{l}\text { Dialysis } \\
\text { (group 2, } n=31)\end{array}$ & $\begin{array}{l}\text { Renal transplant } \\
\text { (group 3, } n=11)\end{array}$ \\
\hline Hydroxychloroquine & $6(86)$ & $29(93)$ & $10(91)$ \\
Darunavir/ritonavir & $4(57)$ & $24(77)$ & $8(73)$ \\
Lopinavir/ritonavir & $2(28)$ & $6(19)$ & $3(27)$ \\
Azithromycin & $5(71)$ & $25(81)$ & $11(100)$ \\
Tocilizumab & $3(43)$ & $5(16)$ & $6(54)$ \\
\hline
\end{tabular}

Data are given in absolute numbers with the percentage in brackets. CKD, chronic kidney disease.

tal population of 49 recovered subjects was $32.4 \pm 12.3$ days (median: 31 days; range 15-58 days). This time span to achieve viral clearance was $38.8 \pm 13.3$ days in group 1 (34 days; range $24-56$ days), $30.8 \pm 12.2$ days in group 2 (median: 30 days; range $15-58$ days), and $32.8 \pm 11.6$ days in group 3 (median of 30 days; range 17-50 days), with no significant intergroup differences ( $p=$ n.s., ANOVA test). Time to negativization did not show any significant correlation with clinical severity at onset, antiviral therapy, or immunosuppressive regimen (transplant group).

Three patients ( 1 in each group) experienced a relapse of COVID-19 infection after 8-13 days from the laboratory recovery. In all the 3 cases, the viral load was significantly lower than that found at the first infection. The following nasopharyngeal swabs showed persistent negativity.

Both IgG and IgM against SARS-CoV-2 were detected in all patients since the first measurement following PCR negativization (range 3-8 days). In the first serological samples, the sample/cutoff (S/CO) index for IgG ranged from 0.93 to 12.12 (mean 7.78) and S/CO index for IgM ranged from 0.2 to 9.4 (mean 1.08). The semiquantitative analysis of SARS-CoV-2 antibodies in the patients who underwent at least 2 serological tests revealed a progressive increase of IgG index up to $37.5 \%$ and a concurrent slow decline of IgM index up to $94 \%$.

IgG remained positive up to 44 days after viral clearance. At the last available determination, IgM were found to be negative in $16 / 49$ patients.

At the time of data collection, patients treated for COVID-19 infection, were in stable clinical conditions and no significant organ damage was reported, except 1 single patient with decompensated renal failure (group 1) who is still dependent on artificial replacement treatment. Other 6 patients of group 1 achieved a partial functional recovery with respect to preinfective period (as indicated by the mild eGFR reduction to $21.6 \pm 9.0 \mathrm{~mL} / \mathrm{min})$. In transplanted patients, the eGFR was also moderately decreased compared to the preinfection period (40.6 \pm 14.05 vs. $47.7 \pm 25.4 \mathrm{~mL} / \mathrm{min}, p=$ n.s.).

\section{Discussion/Conclusion}

SARS-CoV-2 infection is having a tremendous impact on national health systems worldwide. Our data confirm the increased susceptibility to COVID-19 of patients with advanced-stage renal failure [20], under chronic dialysis therapy [21,22] and kidney transplant recipients [23] compared to the general population, as previously reported in Italy and other industrialized countries [8, 24, 25].

The elevated incidence observed especially in hemodialysis patients is mainly related to the frequent access to the hospital, to the duration of individual treatments, implying a prolonged contact with other patients and healthcare personnel, and the necessity of transportation to reach the dialysis center [2]. In our experience, no COVID-19-positive case were found among patients under peritoneal dialysis, thus confirming the advantages related to a home treatment, as already highlighted by recent evidence [26, 27].

The overall mortality of SARS-CoV-2 infection in Italy was $11.6 \%$; a worse outcome is reported in patients with chronic diseases and cardiovascular comorbidity [28]. Consistent with previous reports $[24,25]$, our population showed an elevated death rate $(22.2 \%$ in patients with decompensated renal failure, $38.0 \%$ in dialysis patients, and $31.2 \%$ in transplant recipients), confirming the fragility of chronic nephropathic patients, in spite of the timely therapy against COVID-19 given in most of the cases and the reduction of immunosuppression in transplanted patients. Sadly, the work suffers from the heterogeneity of therapeutic intervention for COVID-19, mainly related to the period in which the study was conducted, the early 
stages of the pandemic outbreak in Italy, when a reference treatment scheme was not well defined yet.

There was also a different diagnostic approach to the infection in the 3 study groups; among hemodialysis patients, the diagnosis was occasional in $10 \%$ of them during the screening for procedure execution not related to COVID-19 (preparation for instrumental examinations and scheduled hospitalizations) and in 15\% for reporting contacts with positive cases. In transplant recipients, managed mainly at home, nasopharyngeal swab sampling was always performed for clinical reasons (fever and respiratory symptoms of variable severity), but this might have led to an underestimation of the incidence of infection.

Our data highlighted a partial worsening of kidney function following COVID-19 infection in patients with advanced chronic renal failure and transplant recipients, but the short observation period prevents us to draw any firm conclusions on this point. The management of chronic nephropathic patients is complex and involves personalized pathways for those who experienced COVID-19. Here, we tried mainly to evaluate the expected time of nasopharyngeal swab negativization in patients who, for different reasons, have an impaired immune response capacity. Even if the evolution of the single case cannot be generalized, a better understanding of the average time span to achieve viral clearance and antibody response in these weak populations might provide useful references for the planning of health-care activities and the logistic needs for therapeutic interventions. Unsurprisingly, our renal patients showed a slower viral clearance (32.4 \pm 12.3 days) than that found in the general population, even in the context of a wide heterogeneity $[11,12,29]$. Moreover, the time to negativization was also longer in the patients diagnosed for COVID-19 due to sporadic cases or due to contact with an ascertained positive case.

In our case history, the time to viral clearance was moderately longer in patients with metabolic decompensation during infection, but we did not find significant differences between 3 groups of patients. The relatively small sample size and the heterogeneity of therapeutic approaches did not allow identification of a correlation between the time to negativization and antiviral therapy or clinical presentation at onset. Similarly, in transplant patients, no correlation with the immunosuppression regimen was possible.

The overall message emerging from our data indicates a delayed viral clearance of SARS-CoV-2 and can be interpreted in view of the cellular and humoral immunode- pression typical of this population [7, 30, 31]. Comparably to other coronaviruses, SARS-CoV-2 has been proven to induce a T-lymphocyte-mediated immune response, but this protective mechanism might be suppressed in patients with lymphopenia, including those under chronic hemodialysis and renal transplant recipients $[32,33]$.

A striking finding was the discovery of 3 reinfections in patients who, in a time span ranging between 8 and 13 days following laboratory recovery, showed PCR positivity. It must be specified that swab sampling was performed due to subjective symptoms (fever or high respiratory tract inflammation), not through systematic search, and this might have possibly underestimated the rate of reinfection. It is known that the nasopharyngeal swab test has some limitations: the sensitivity is about $80 \%$, implying that in up to $20-30 \%$ the test can produce false negatives, mainly related to too early timing of the sampling or human error in collecting adequate material. This limited accuracy suggests some caution before the complete reintroduction of patients with previous infection into the open-space sections of hemodialysis or in transplant follow-up surgeries. Applying a principle of extreme prudence in order to protect other fragile patients, the ones recovered from COVID-19 should be managed in filter zones for a period of 15-20 days. The development of rapid tests and/or highly accurate assays able to define the presence of the virus and not only of parts of the genome may be helpful in the near future.

Serological tests represent a useful integration of nasopharyngeal swab data. In our experience, all the patients treated for the infection have developed an antibody response, with a progressive increase and subsequent stabilization of IgG associated with a slow reduction or disappearance of IgM. The interpretation of serological tests remains complex and must be integrated into the clinical context and with other laboratory investigations. The assay used for antibody detection has a good sensitivity and specificity. The progressive reduction/disappearing of IgM is in line with the physiological immunological response. The finding of a prolonged persistence of $\operatorname{IgM}$ at the end of the observation period in $67 \%$ of our population studied is not a criterion of infection in progress. These data should be confirmed in a larger population, a wider range of cases, also keeping in mind that the detection of IgM has a lower specificity (high false-positive rate) due to increased cross-reactivity with other coronaviruses $[34,35]$. The serological study requires a prolonged monitoring to verify the antibody changes over time and their persistence. The titration of IgG antibodies to identify a threshold level that confers an effective pro- 
tection against the disease may represent a valuable tool for the recognition of immune subjects and for the possible management of vaccination programs. These first data on our renal patients show an overall satisfying antibody response capacity.

However, this study does not analyze the prognostic factors for COVID-19 recovery, which is still a matter of debate in current research. But to the best of our knowledge, this is the first investigation focused on the time to reach viral clearance and the development of specific antibodies against SARS-CoV-2 in different groups of chronic nephropathic patients. From an epidemiological perspective, a systematic screening of this weak population is desirable, regardless of an acute symptomatology and/or a diagnosis of infection. The limitations of the study are related to its retrospective nature, based on the analysis of laboratory data, performed in a clinical context of a critical health emergency due to the peak of pandemic outbreak that did not allow a uniform assay schedule. In particular, it was impossible to carry out the serology testing at programmed time points referred to the onset of infection (first positive swab) and to the moment of viral clearance ( 2 consecutive negative swabs). Another weakness is represented by the small sample size and the relatively short observation period. Serological investigations were performed after nasopharyngeal swab negativization, and this does not consent a full evaluation of antibody kinetics since the initial stages of their production. Furthermore, since the observation period was February-April 2020 during the most dramatic stage of pandemic, our efforts at that time were focused on our nephropathic patients known to have an impaired immune response, and we did not include a control group of subjects hospitalized for COVID-19 with normal renal function.

The experience gained during the pandemic leads to some considerations on the structural reorganization of spaces and access/exit routes of the Nephrology and Hemodialysis Units. The importance of prophylaxis actions that must be constantly maintained remains unquestionable; obviously, the workload of the different structures is amplified, due to the strict necessity of adequate filter procedures.

In conclusion, COVID-19 infection is a significant clinical problem in nephropathic patients and in kidney transplant recipients. Our findings reveal a delayed viral clearance in this fragile population, while there is a satisfying ability to produce specific antibodies. In a context still characterized by many clinical and epidemiological critical points, not fully clarified yet, a better understand- ing of the immune response in patients with unpaired renal function can represent a useful basis for further prospective research and in the management of vaccination programs. In weak populations with a high risk of morbidity and mortality, it is necessary to implement all the preventive measures to minimize the risk of COVID-19 infection and to identify positive patients at an early stage.

\section{Statement of Ethics}

Ethics Committee approval and informed consent were waived due to the observational nature of the study. The study followed the principles of the Declaration of Helsinki.

\section{Conflict of Interest Statement}

The authors have no conflicts of interest to declare.

\section{Funding Sources}

No funding was received in support of the present work.

\section{Author Contributions}

P.F.B.: data collection and manuscript drafting; M.C.: statistical analysis, manuscript preparation, editing, and revision; A.S., M.D.L., B.S., M.N., and V.M.: patients' management, sample, and data collection; S.S., G.D., and V.S.: microbiology testing and validation; A.B., A.R., and E.M.: main supervision on patients' management, sample, and data collection; P.M., G.L.M, and G.M: conceptualization and main supervision.

\section{References}

1 Al-Tawfiq JA, Hinedi K, Ghandour J, Khairalla $\mathrm{H}$, Musleh S, Ujayli A, et al. Middle East respiratory syndrome coronavirus: a casecontrol study of hospitalized patients. Clin Infect Dis. 2014 Jul 15;59(2):160-5.

2 Rubin R. Finding ways to reduce coronavirus exposure during dialysis. JAMA. 2020 May 26;323(20):1993-5

3 Johnson KM, Belfer JJ, Peterson GR, Boelkins MR, Dumkow LE. Managing COVID-19 in renal transplant recipients: a review of recent literature and case supporting corticosteroidsparing Immunosuppression. Pharmacotherapy. 2020 Jun;40(6):517-24.

4 Xiao Y, Qian K, Luo Y, Chen S, Lu M, Wang $\mathrm{G}$, et al. Severe acute respiratory syndrome coronavirus 2 infection in renal failure patients: a potential covert source of infection. Eur Urol. 2020 Aug;78(2):298-9. 
5 Cheng Y, Wang W, Wu L, Cai G. SARS-CoV2-related kidney injury: current concern and challenges. SN Compr Clin Med. 2020 Sep 23: 1-10.

6 Loganathan S, Kuppusamy M, Wankhar W, Gurugubelli KR, Mahadevappa VH, Lepcha $\mathrm{L}$, et al. Angiotensin-converting enzyme 2 (ACE2): COVID 19 gate way to multiple organ failure syndromes. Respir Physiol Neurobiol. 2021 Sep 18;283:103548.

7 Guan WJ, Ni ZY, Hu Y, Liang WH, Ou CQ, $\mathrm{He} \mathrm{XX}$, et al. Clinical characteristics of coronavirus disease 2019 in China. N Engl J Med. 2020 Apr 30;382(18):1708-20.

8 Quintaliani G, Reboldi G, Di Napoli A, Nordio M, Limido A, Aucella F, et al. Exposure to novel coronavirus in patients on renal replacement therapy during the exponential phase of COVID-19 pandemic: survey of the Italian society of nephrology. J Nephrol. 2020 Aug;33(4):725-36.

9 Gautret P, Lagier JC, Parola P, Hoang VT, Meddeb L, Mailhe M, et al. Hydroxychloroquine and azithromycin as a treatment of $\mathrm{CO}$ VID-19: results of an open-label non-randomized clinical trial. Int $J$ Antimicrob Agents. 2020 Jul;56(1):105949.

10 Molina JM, Delaugerre C, Le Goff J, Mela-Lima B, Ponscarme D, Goldwirt L, et al. No evidence of rapid antiviral clearance or clinical benefit with the combination of hydroxychloroquine and azithromycin in patients with severe COVID-19 infection. Med Mal Infect. 2020 Jun;50(4):384.

11 Yuan J, Zou R, Zeng L, Kou S, Lan J, Li X, et al. The correlation between viral clearance and biochemical outcomes of 94 COVID-19 infected discharged patients. Inflamm Res. 2020 Jun;69(6):599-606.

12 Chang D, Mo G, Yuan X, Tao Y, Peng X, Wang FS, et al. Time kinetics of viral clearance and resolution of symptoms in novel coronavirus infection. Am J Respir Crit Care Med. 2020 May 1;201(9):1150-2.

13 Ling Y, Xu SB, Lin YX, Tian D, Zhu ZQ, Dai $\mathrm{FH}$, et al. Persistence and clearance of viral RNA in 2019 novel coronavirus disease rehabilitation patients. Chin Med J. 2020 May 5; 133(9):1039-43.

14 Ikizler TA. COVID-19 and dialysis units: what do we know now and what should we do? Am J Kidney Dis. 2020 Jul;76(1):1-3.

15 Levey AS, Stevens LA. Estimating GFR using the CKD epidemiology collaboration (CKD-
EPI) creatinine equation: more accurate GFR estimates, lower CKD prevalence estimates, and better risk predictions. Am J Kidney Dis. 2010 Apr;55(4):622-7.

16 Istituto Superiore di Sanità Raccomandazioni per il corretto prelievo, conservazione e analisi sul tampone oro/nasofaringeo per la diagnosi di COVID-19. Versione 2 del 29 maggio 2020. Gruppo di Lavoro ISS Diagnostica e sorveglianza microbiologica COVID-19: aspetti di analisi molecolare e sierologica 2020, ii, 7 p. Rapporti ISS COVID-19 no. $11 / 2020$.

17 World Health Organization. Clinical management of severe acute respiratory infection when Novel coronavirus (2019-nCoV) infection is suspected: interim guidance. 2020 Jan 28 (WHO/nCoVClinical/2020.3).

18 Antonelli G, Stefani S, Pistello M. SARSCoV-2 diagnostics: some reflections on current assays. Diagn Microbiol Infect Dis. 2021 Oct 7;99(2):115237.

19 Ratnam S, Tipples G, Head C, Fauvel M, Fearon M, Ward BJ. Performance of indirect immunoglobulin $\mathrm{M}$ (IgM) serology tests and IgM capture assays for laboratory diagnosis of measles. J Clin Microbiol. 2000 Jan;38(1):99104.

20 Trujillo H, Caravaca-Fontán F, Sevillano Á, Gutiérrez E, Caro J, Gutiérrez E, et al. SARS$\mathrm{CoV}-2$ infection in hospitalized patients with kidney disease. Kidney Int Rep. 2020 May 1; 5(6):905-9.

21 Weiss S, Bhat P, Del Pilar Fernandez M, Bhat JG, Coritsidis GN. COVID-19 infection in ESKD: findings from a prospective disease surveillance program at dialysis facilities in New York City and Long Island. J Am Soc Nephrol. 2020 Nov;31(11):2517-21.

22 Hsu CM, Weiner DE. COVID-19 in dialysis patients: outlasting and outsmarting a pandemic. Kidney Int. 2020 Dec;98(6):1402-4.

23 Azzi Y, Parides M, Alani O, Loarte-Campos $\mathrm{P}$, Bartash R, Forest S, et al. COVID-19 infection in kidney transplant recipients at the epicenter of pandemics. Kidney Int. 2020 Dec; 98(6): 1559-67.

24 Kant S, Menez SP, Hanouneh M, Fine DM, Crews DC, Brennan DC, et al. The COVID-19 nephrology compendium: AKI, CKD, ESKD and transplantation. BMC Nephrol. 2020 Oct 27;21(1):449.

25 Cota LB, Pedrosa AL, de Brito SBCS, Fróes ACF, de Carvalho ST, Fonseca GG, et al. CO-
VID-19 and renal diseases: an update. Curr Drug Targets. 01 Jan 2021;22(1):52-67.

26 Brown EA, Perl J. Increasing peritoneal dialysis use in response to the COVID-19 pandemic: will it go viral? J Am Soc Nephrol. 2020 Sep; 31(9):1928-30.

27 Srivitana V, Liu F, Levine DM, Kalloo SD. Early use of telehealth in home dialysis during the COVID-19 pandemic in New York city. Kidney. 2020;360(1):524-6.

28 Flaherty GT, Hession P, Liew CH, Lim BCW, Leong TK, Lim V, et al. COVID-19 in adult patients with pre-existing chronic cardiac, respiratory and metabolic disease: a critical literature review with clinical recommendations. Trop Dis Travel Med Vaccines. 2020 Aug 28;6:16.

29 Mosconi G, Spazzoli A, Bruno PF, Angelini ML, Cristino S, Lifrieri MF, et al. [Resilience in COVID-19 times: general considerations on the recovery of a 93-year-old patient on haemodialysis treatment]. G Ital Nefrol. 2020 Oct 5;37(5):2020-vol5.

30 Merli M, Pasulo L, Perricone G, Travi G, Rossotti R, Colombo VG, et al. Impact of immunosuppressive therapy on the severity of COVID-19 in solid organ transplant recipients. J Infect. 2020 Oct 27;82(3):414-451.

31 Arslan H, Musabak U, Ayvazoglu Soy EH, Kurt Azap O, Sayin B, Akcay S, et al. Incidence and immunologic analysis of coronavirus disease (COVID-19) in hemodialysis patients:a single-center experience. Exp Clin Transplant. 2020 Jun;18(3):275-83.

32 Raoult D, Zumla A, Locatelli F, Ippolito G, Kroemer G. Coronavirus infections: epidemiological, clinical and immunological features and hypotheses. Cell Stress. 2020 Mar 2;4(4): 66-75.

33 Wang D, Hu B, Hu C, Zhu F, Liu X, Zhang J, et al. Clinical characteristics of 138 hospitalized patients with 2019 novel coronavirus-infected pneumonia in Wuhan, China. JAMA. 2020 Mar 17;323(11):1061-9.

34 Wang AX, Quintero Cardona O, Ho DY, Busque S, Lenihan CR. Influence of immunosuppression on seroconversion against SARSCoV-2 in two kidney transplant recipients. Transpl Infect Dis. 2021 Jul 23;23(1):e13423.

35 Tang MS, Hock KG, Logsdon NM, Hayes JE, Gronowski AM, Anderson NW, et al. Clinical performance of two SARS-CoV-2 serologic assays. Clin Chem. 2020 Aug 1;66(8):1055-62. 